\title{
LEFT ORE PRINCIPAL RIGHT IDEAL DOMAINS
}

\author{
RAYMOND A. BEAUREGARD
}

(Communicated by Donald Passman)

\begin{abstract}
The rings referred to in the title of this paper have long been conjectured to be principal left ideal domains. In a recent paper [6] Cohn and Schofield have produced examples (of simple rings) showing that this is not the case. As a result, interest in this type of ring has deepened (see [5], for example, where these rings are referred to as right principal Bezout domains). Our main purpose here is to prove that such rings are either principal left ideal domains or left and right primitive rings.
\end{abstract}

All rings below have unity and have no proper zero-divisors. A principal right ideal domain (PRI domain) is a ring in which each right ideal is a principal right ideal. Such a ring is an example of a weak Bezout domain or 2-fir, i.e., a ring in which the sum and intersection of any two principal right ideals is again principal whenever the intersection is nonzero. In addition, a PRI domain is a right Ore domain, i.e., a ring in which each nonzero principal right ideal is right large in the sense that it has nonzero intersection with each nonzero right ideal. A right Ore 2fir is a right Bezout domain. The concept of a 2-fir is left-right symmetric; however a right Bezout domain is left Bezout iff it is also left Ore. We refer to [4] for the proof of these well-known results.

A nonzero nonunit in a ring $R$ is an atom (also called prime or irreducible) if it has no proper factors. An element which is either a unit or a product of atoms is said to be finite dimensional or simply finite. A ring is said to be atomic if each of its nonzero elements is finite. In a 2 -fir this condition is equivalent to the acc for principal right and also principal left ideals. In a PRI domain only the right acc need hold, assuring that each nonzero nonunit has an atomic left factor. Also recall that in a 2-fir, atomic factorizations are unique up to order of factors and similarity.

The following proposition generalizes the well-known fact that an atomic left Ore PRI domain is a PLI domain.

PROPOSITION 1. Let $R$ be a 2-fir. If $I$ is a left ideal of $R$ containing a finite element $a$ which is left-large (i.e., $R a \cap R x \neq 0$ for all $0 \neq x \in R$ ), then $I$ is a principal left ideal.

PROOF. Let $a_{1}=a$. If $R a_{1} \neq I$ choose $a_{2} \in I \backslash R a_{1}$. Inductively, let $I_{n}=$ $R a_{1}+\cdots+R a_{n}$; if $I \neq I_{n}$ choose $a_{n+1}$ in $I \backslash I_{n}$. Since $a_{1}$ is large, each $I_{n}$ must be a principal left ideal and since $a_{1}$ is finite the chain $I_{n}$ must itself be finite. Thus $I$ is a principal left ideal.

Received by the editors December 9, 1986.

1980 Mathematics Subject Classification (1985 Revision). Primary 16A02.

Key words and phrases. Principal ideal domain, Ore domain, Bezout domain, invariant element, bounded element, primitive ring. 
A nonzero element $a$ in a ring $R$ is right (left) invariant if $a R(R a)$ is a two-sided ideal; if both conditions hold, that is, if $a R=R a$, then $a$ is invariant.

PROPOSITION 2. In any ring $R$, if $a$ is right invariant then every factor of $a$ is a left factor; if $a$ is invariant then every proper factor of $a$ is a proper left factor.

ProOF. Assume $b$ is a factor of $a$, say $a=r b s$ in $R$. The right invariance of $a$ gives rise to an endomorphism $\sigma$ of $R$ given by $r a=a r^{\sigma}$. Then $a=b s r^{\sigma}$ follows easily. If $a$ is invariant, then $\sigma$ is an automorphism and so if $r$ is a nonunit then so is $r^{\sigma}$.

PROPOSITION 3. In any ring $R$, if $a=c b$ with $c$ left invariant and $a$ right invariant, then $b$ is right invariant.

PROOF. For any $r \in R$, if $c r=r^{\prime} c$ and $r^{\prime} a=a r^{\prime \prime}$ then $r b=b r^{\prime \prime}$ so that $R b \subseteq b R$.

Let $R$ be a PRI domain. A nonzero element $b$ in $R$ is said to be right bounded if $b R$ contains a nonzero two-sided ideal; the largest such ideal is denoted $b^{*} R$ and $b^{*}$ is the right bound of $b$. If $R$ is a left Ore PRI domain, a similar statement holds for left bounded elements and the left bound of $b$ is denoted $b^{\sharp}$ if it exists. This follows from [2, Theorem 2.2] and the fact that the family of principal left ideals of $R$ is closed under intersections. This last property for $R$ is an immediate consequence of the restricted dcc for principal left ideals which is equivalent to the acc for principal right ideals [2, p. 253].

From now on we assume that $R$ is a left Ore PRI domain. Suppose $b \in R$ has left bound $b^{\sharp}$. Then since $R b^{\sharp}$ is a right ideal, $R b^{\sharp}=x R$ for some $x \in R$; from this $R b^{\sharp}=b^{\sharp} R$ follows easily. In addition, the left-right analog of Theorem 3.2 of [2] applies and shows that if $p$ is an atom which is left bounded then $p^{\sharp}$ is also finite. Thus the left bound of an atom is finite invariant. Using this fact we can prove the following result.

PROPOSITION 4. Let $R$ be a left Ore PRI domain. If $a R=R a$, then a is finite.

PROOF. Suppose the result is false and choose $a R$ maximal in $\{x R \mid x R=R x$, $x$ not finite in $R$ \}. Let $p$ be an atomic (left) factor of $a$. Then $p$ is bounded by $a$ and so has left bound $p^{\sharp}$ which is finite invariant by the remark above and divides $a$. Proposition 3 and its left-right analog applied to an equation $a=y p^{\sharp}$ shows $y$ to be invariant. Thus $a R \subsetneq y R$ and $y$ is not finite because $p^{\sharp}$ is finite. We have a contradiction to the way $a R$ is chosen and the proof is complete.

Proposition 4 and the remarks preceding it give us the following.

COROllary 5. Let $R$ be a left Ore PRI domain. If $b \in R$ has left bound $b^{\sharp}$ then $b^{\sharp}$ is finite invariant.

A ring $R$ is said to be left (right) bounded if each of its elements is left (right) bounded.

Corollary 6 (CF. [3]). A left bounded PRI domain is a PLI domain.

ProOF. If $R$ is left bounded, then $R$ is clearly left Ore. Proposition 1, together with Corollary 5 , shows that each left ideal of $R$ is principal. 
THEOREM 7. Let $R$ be a left Ore PRI domain. If each atom of $R$ is left bounded, then $R$ is left bounded.

PROOF. The set of all left bounded elements of $R$ is multiplicatively closed [2, Theorem 2.7]. Thus each finite element of $R$ is left bounded. Conversely each left bounded element of $R$ is finite by Corollary 5. Suppose that $R$ is not left bounded. Then we may choose $b R$ maximal such that $b$ is infinite ( $b$ is an infinite prime of [1]). We obtain a contradiction using an argument like that of [5, Proposition 6.1]. Let $p$ be an atomic left factor of $b$ and let $p^{\sharp}$ be its (finite invariant) left bound. The lcrm of $b$ and $p^{\sharp}$ may be written

$$
m=b x=p^{\sharp} y \text {. }
$$

If $b R+p^{\sharp} R=d R$ then $b=d b_{1}, p^{\sharp}=d p_{1}$ and $b_{1} \sim y, p_{1} \sim x$ (the symbol $\sim$ stands for similarity). Note that $p$ is a left factor of $p^{\sharp}$ by Proposition 2 . Thus $x$ is finite with length less than that of $p^{\sharp}$ and $y$ is infinite. The invariance of $p^{\sharp}$ defines an automorphism $\sigma$ of $R$ given by $r p^{\sharp}=p^{\sharp} r^{\sigma}$. We may write $b p^{\sharp}=p^{\sharp} b^{\sigma}$ in the form $m z$ for some $z \in R$. Comparing with the displayed equation we have $b^{\sigma}=y z, p^{\sharp}=x z$ and $z$ is a finite nonunit while $y$ is infinite. Writing $\theta=\sigma^{-1}$ we have $b=y^{\theta} z^{\theta}$, contradicting the maximal way $b R$ is chosen.

THEOREM 8. Let $R$ be a left Ore PRI domain. Either $R$ is a PLI domain or $R$ is a left primitive ring.

ProOF. Suppose that $R$ is not a PLI domain. Then $R$ is not left bounded by Corollary 6. Thus we may select an atom $p$ in $R$ such that $R p$ contains no nonzero two-sided ideal. If $M$ is any left ideal of $R$ containing $R p$, then Proposition 1 shows that $M$ must be a principal left ideal (containing the atom $p$ ) and so $R p$ is maximal as left ideal. Thus $R / R p$ is a simple faithful left $R$-module.

COROLlaRY 9 (CF. [5]). If $R$ is a left Ore PRI domain, then either $R$ is a $P L I$ domain or the Jacobson radical of $R$ is zero.

Since a local primitive ring is a (skew) field we obtain the following well-known result as a corollary.

COROllary 10. A local left-Ore PRI domain is a PLI domain.

We wish to establish Theorem 8 but with right primitive in place of left primitive. To do this we shall use the following result whose proof is given in [5] (compare with Corollary 5).

Proposition 11 (CF. [5, Proposition 6.2]). Let $R$ be a left Ore PRI domain. If $p$ is an atom of $R$ having right bound $p^{*}$, then $p^{*}$ is finite invariant.

Using this result we can prove the following.

THEOREM 12. Let $R$ be a left Ore PRI domain. Every nonzero two-sided ideal of $R$ is a principal left ideal generated by a finite invariant element.

Proof. Note that any two-sided ideal $I \neq 0$ of $R$ has the form $I=a R$ and is a principal left ideal iff $a$ is finite by Propositions 1 and 4. Suppose the theorem we wish to prove is false. Choose $a R$ maximal in $\{x R \mid R x \subseteq x R$ and $x$ is not finite $\}$. Let $p$ be an atomic left factor of $a$ and $p^{*}$ its invariant right bound so that 
$a R \subseteq p^{*} R$. We write $a=p^{*} b$ for some infinite $b$ in $R$. Applying Proposition 3 we see that $b$ is right invariant. Since $p^{*}$ is invariant we have $a=c p^{*}$ and $c$ (like $b$ ) is also right invariant and infinite. Thus $a R \subsetneq c R$ contradicting the choice of $a$.

As a consequence of Theorem 12 we see that for $b$ in $R$, the left bound $b^{\sharp}$ exists iff the right bound $b^{*}$ exists, in which case $b^{*} R=R b^{\sharp}$. Thus we obtain the following improvement of Corollary 6 answering a question raised in [3].

COROLlaRY 13. Let $R$ be a left Ore PRI domain. If $R$ is either left or right bounded, then $R$ is a PLI domain.

In addition we have the following analog of Theorem 8.

THEOREM 14. Let $R$ be a left Ore PRI domain. Either $R$ is a PLI domain or $R$ is a right primitive ring.

PrOOF. If $R$ is not a PLI domain then $R$ is not left bounded. Thus we have an atom $p$ in $R$ which is not left bounded and hence not right bounded. Then $R / p R$ is a simple faithful right $R$-module.

If $R$ is a primitive PRI domain, then $R$ will be simple if all atoms of $R$ are similar. For, if $M=a R$ is a maximal ideal of $R$ and $a \neq 0$, then $a$ is the right bound of some atom $p$. But then $a R=\bigcap_{q} q R$ where the intersection is over all atoms of $R$ by [2, Theorem 2.2], that is $M=a R$ is the Jacobson radical of $R$. Thus $M=0$.

The examples given in [6] of left Ore PRI domains that are not PLI domains are simple rings. For examples of primitive PLI-PRI domains that are not simple we mention the skew polynomial ring $F[x, \sigma]$, where $\sigma$ is an automorphism of the field $F$ of infinite order (cf. [7, p. 22]). To see what can happen in a PRI domain that is not left Ore see Example 3.9 of [2].

ADDENDUM. This paper was written before the appearance of [5] in revised form. Hence Proposition 4 follows from [5, Proposition 6.3] and Theorem 12 is essentially [5, Theorem 6.4].

\section{REFERENCES}

1. R. A. Beauregard, Infinite primes and unique factorization in a principal right ideal domain, Trans. Amer. Math. Soc. 141 (1969), 245-253.

2. - Right bounded factors in an LCM domain, Trans. Amer. Math. Soc. 200 (1974), 251-266.

3. $\_$, Left and right invariance in an integral domain, Proc. Amer. Math. Soc. 67 (1977), 201-205.

4. P. M. Cohn, Free rings and their relations, 2nd ed., Academic Press, London, New York, 1985.

5. __ Right principal Bezout domains, J. London Math. Soc. (2) 35 (1987), 251-262.

6. P. M. Cohn and A. H. Schofield, Two examples of principal ideal domains, Bull. London Math. Soc. 17 (1985), 25-28.

7. N. Jacobson, Structure of rings (revised), Amer. Math. Soc. Colloq Publ., vol. 37, Providence, R.I., 1964.

DEPARTMENT OF MATHEMATICS, UNIVERSITY OF RHODE ISLAND, KingSTON, RHODE ISLAND 02881 